\title{
ENERGETICS, COLLIMATION AND PROPAGATION OF GALACTIC PROTOSTELLAR OUTFLOWS
}

\author{
Views and Perspectives
}

\author{
MAX CAMENZIND
}

Landessternwarte Königstuhl, D-69117 Heidelberg, Germany

\begin{abstract}
Formation of jets in low-mass protostellar objects and young pre-main sequence stars is ultimately related to the existence of some gaseous disk around a rapidly rotating central object. This configuration has deep parallels to extragalactic systems such as radio galaxies and quasars. Rotating black holes are still thought to be the prime-mover behind the activity detected in centers of galaxies, while, in the case of protostellar jets, rapidly rotating stars and disks are responsible for the ejection of bipolar outflows. In both cases, magnetic fields are invoked for the acceleration, the collimation and propagation of these outflows. The ultimate rooting of these fields is still under debate. We discuss models where winds injected into rapidly rotating magnetospheres of the central object drive the outflows. From these considerations it follows that the jets of young stellar objects can only be produced magnetically and that their progagation is determined by their magnetic properties. Such jets have low Mach numbers $\simeq 2$ and their instabilities are dominated by the pinch mode. Knots closest to the source are attributed to compression by the time-dependent pinches. Multiple bow shocks occur on longer time-scales (a few thousand years) and are attributed to variations in the magnetospheric structure of the star, or the disk.
\end{abstract}

\section{Introduction}

It is now known for a long time that young low-mass stars are capable of driving highly collimated bipolar outflows with velocities of several hundred $\mathrm{km} \mathrm{s}^{-1}$. The association of many outflows with stars deeply imbedded in their molecular cloud cores suggests that the energetic winds are intrinsic to the star formation process itself. In fact, they may provide the only means 
for a collapsing and rotating object to shed sufficient angular momentum (as already proposed by Hartmann \& MacGregor in 1982). An additional impact of these winds consists in reversing the infall of core material and thereby determining the final mass of the star.

In addition to embedded sources, energetic winds are also observed from optically visible pre-main sequence stars with ages of the order of a few million years, or less. Unlike these classical T Tauri stars (cTTS), the embedded infrared sources (EIR) are observed over a wide range of bolometric luminosities $\left(0.1\right.$ to $\left.10^{5} L_{\odot}\right)$, indicating that mass outflows are driven by many types of masses. The energetic outflows from the EIR are primarily traced by swept-up shells of high velocity molecular gas (tens of $\mathrm{km} \mathrm{s}^{-1}$ ) with structures of bipolar topology and extending up to several parsecs. In a few cases, the driving wind interior to the molecular shells has been detected as a very high speed neutral wind with velocities of the order of a few hundred $\mathrm{km} \mathrm{s}^{-1}$. The determination of the mechanical luminosities and momentum fluxes from molecular outflows has been given by Lada (1985; see also Lada \& Fich, 1996).

The most spectacular and also most probably youngest outflows have been found in near-infrared surveys (McCaughrean et al., 1994; Zinnecker et al., 1996). These jets quite often show inner knots and signs of swept-up ambient molecular gas.

The most popular theoretical ideas for the ultimate source of momentum and energy in these outflow sources are based on the existence of winds driven away by magnetic fields of the surrounding accretion disk (Blandford \& Payne 1982; Pelletier \& Pudritz, 1992; Ferreira \& Pelletier, 1995; Ouyed et al., 1997). All these models invoke a dipolar magnetic structure in the disk which is essentially advected inwards from the surrounding molecular core. Accretion disks of low-mass protostellar objects are, however, in most cases convective such that the postulated magnetic structures are unstable due to dynamo action (Camenzind, 1990). The normal modes excited by geometrically thin disks are of the quadrupolar type (Camenzind, 1990; Stepinski \& Levy, 1991; Rüdiger et al., 1995; Camenzind \& Lesch, 1994), and therefore unfavourable for driving efficiently disk winds.

Since the protostar itself is in a fully convective state, dynamo operation is unavoidable and leads to surface field strengths of the order of one kilo-Gauss. This seems to be quite natural for a rapidly rotating protostellar object. Rapid rotation together with unipolar induction generate a Poynting luminosity of the surface of the protostar which is in fact exactly of the same order of magnitude as the kinetic luminosity observed in protostellar outflows. This fact indicates that not the gravitational potential of the star, but the rotational energy of rapidly rotating objects is the source of the kinetic energy observed in the outflows. It is a consequence of the 
conservation law for the total energy consisting of magnetic energy and kinetic energy along a given flux surface of a rotating magnetosphere.

In the next Sect. we discuss possible jet formation scenarios from the point of view of rapidly rotating magnetospheres. One of the key questions for magnetically driven outflows is the origin of the magnetic fields in the protostellar environment. We discuss the dynamo equations for convective stars and disks. It turns out that the characteristic dynamo time for protostars is of the order of a few hundred years. Seed fields left over from the protostellar collapse will therefore be rapidly destroyed and restructured in a short time most probably into a dipolar structure inside the star and some complicated quadrupolar structure along the disk. Though the outcome of these dynamical systems is not known - and will remain unknown for the next years - we speculate that the stellar field is indeed a dipolar configuration.

Such a dipolar structure will strongly interact with the ambient disk. Accretion towards the stellar surface is channeled into an accretion column, and strong winds will be driven away from the polar cap regions. These magnetized winds are then collimated into a cylindrical shape at a distance of about one hundred astronomical units, corresponding to about 10000 stellar radii. In Sect. 3 we summarize the basic facts of axisymmetric MHD which show the existence of self-collimated outflows. It is interesting that such structures naturally occur when the electric fields, generated by the rapid rotation of the magnetosphere beyond the light cylinder, are taken into account in the force-balance equation. The light cylinder is in fact at a radius of $100 \mathrm{AU}$ for a rotation period of 3 days. Since electric fields are neglected in the usual treatment of magnetized winds, people missed the existence of such self-collimated solutions. The resulting jet outflows have a very simple structure, as discussed in Sect. 4 . The magnetic field is predominantly toroidal with a field strength of the order of a few milliGauss, corresponding to a fast magnetosonic speed of about $100 \mathrm{~km} / \mathrm{s}$ for the typical density expected at $100 \mathrm{AU}$ in the jet. This means that the sonic speed is completely irrelevant for the dynamics of the jet. In fact, these jets have a low plasma $\beta=P_{\text {gas }} / P_{m} \simeq 10^{-2}$. Only magnetic and electric fields are responsible for the pressure-equilibrium across the jet. This also means that the gas flowing in the jet at a speed of $\simeq 300 \mathrm{~km} / \mathrm{s}$ has a low magnetosonic Mach number of the order of $2-3$.

Traditionally, the propagation of collimated flows through the interstellar medium has been described in terms of hydrodynamical flows (Stone \& Norman, 1993; Suttner et al., 1996). This requires a high sonic Mach number $M \geq 50$ and means therefore practically no interaction with the ambient medium. The regular spacing of knots can therefore not be explained in terms of internal shocks. The fact that jets are supermagnetosonic opens up 
the possiblity that the interaction with the ambient medium is in fact much stronger than in hydro-jets. The most dangerous mode excited for magnetic jets is the fundamental pinch and kink mode (Appl \& Camenzind, 1992). This could be responsible for mixing up with the ambient medium leading in this way e.g. to regularly spaced $\mathrm{H}_{2}$-knots as observed quite recently. It also means that the bow shock structure is much more complicated than in simple hydro-jets. Therefore, also the interpretation of the Herbig-Haro objects has to be carefully re-analyzed.

\section{Jet Formation Scenarios}

Observationally, there are various constraints on the typical parameters for jet formation. HST data (Ray et al., 1996; Stapelfeldt et al., 1995) support models in which the outflow is initially poorly focused and then collimated into a cylindrical structure with diameters of at least of order of a few tens of AU. This is the most important information concerning the overall structure of young stellar jet formation. These scales correspond typically to a few thousand stellar radii. Jets are not pencil-like outflows from the inner edge of accretion disks, these are wide outflows, probably initially of spherical shape, until the flows are suddenly collimated into a cylindrical flow structure (Fig. 1). These geometric constraints are the most severe restrictions on jet formation models. This also means severe implications on the dynamic range of numerical jet formation. In the framework of magnetohydrodynamic (MHD) models this implies solar wind type outflows from the inner part of the star-disk system with a collimation mechanism operating preferentially on the scale of a few thousand stellar radii.

Rapidly rotating objects have been proposed to be the ultimate sources of collimated outflows, once they carry a sufficiently strong magnetosphere. Since Keplerian disks represent the most extreme rotational states, they are prime candidates for jet drivers (Pudritz \& Norman, 1986; Pelletier \& Pudritz, 1993; Ferreira et al., 1995). The crucial question here is whether the disk is able to maintain a dipolar magnetic structure.

\subsection{JETS AS COLLIMATED DISK WINDS}

We consider the interaction of an inward flow of material inside a disk with velocity $\vec{v}$ and the magnetic field in the disk. The disks near protostars are probably heavily convective and therefore prone to dynamo action. Advection and convective motion lead to a source for poloidal magnetic fields $\vec{B}_{p}=\left(\nabla \Psi \times \vec{e}_{\phi}\right) / R$ given in terms of the flux function $\Psi=R A_{\phi}$ (or the 


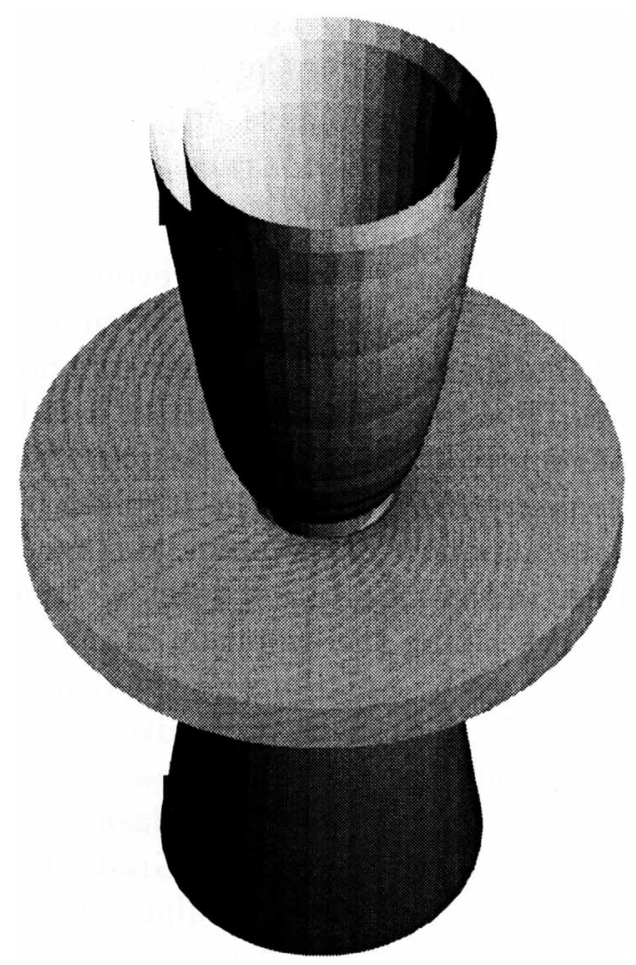

Figure 1. A schematic view on outflows driven by dipolar magnetospheres of rapidly rotating disks and stars. Rotating field lines form axisymmetric magnetic surfaces centered on the rotational axis. Plasma is streaming along these surfaces which are collimated at a certain radius into essentially a cylindrical shape. For protostellar objects, this collimation radius is observed to be about $50 \mathrm{AU}$, disks extend to a few $100 \mathrm{AU}$. The plasma is in force-balance perpendicular to these surfaces.

vector potential $A_{\phi}$ )

$$
\frac{\partial \Psi}{\partial t}+\vec{v} \cdot \nabla \Psi-\eta R^{2} \nabla \cdot\left[\frac{1}{R^{2}} \nabla \Psi\right]=\alpha R B_{\phi}
$$

Poloidal magnetic flux $\Psi$ undergoes a time-evolution due to the advection of the flux by means of the accretion process (with velocity $\vec{v}$ ) and diffusion due to turbulent processes, given by the magnetic diffusivity $\eta \simeq l_{c} v_{c} / 3$ with convective velocity $v_{c}$ on scales $l_{c} \leq H$. Regeneration out of toroidal magnetic fields $B_{\phi}$, or its current function $R B_{\phi}$, occurs due to the dynamo 
effect represented by the function $\alpha \simeq v_{c}$. This toroidal field itself undergoes a similar time-evolution, written here for the current function $I=R B_{\phi}$,

$$
\frac{\partial I}{\partial t}+R^{2} \nabla \cdot\left[\frac{\vec{v}}{R^{2}} I\right]-R^{2} \nabla \cdot\left[\frac{\eta}{R^{2}} \nabla I\right]=R^{2} \vec{B}_{p} \cdot \nabla \Omega .
$$

Differential rotation in disks mainly drives the evolution of the toroidal component, winding it up to equipartition field strengths. The magnetic diffusivity $\eta$ is in general $R$-dependent and can therefore not be taken out of the diffusive term. When dynamo action is absent, $\alpha=0$, the poloidal field structure evolves independently of the toroidal field and is only determined by initial and outer boundary conditions. The toroidal field is essentially determined by the radial component of the poloidal magnetic field. If there is no bending of field lines in the disk, toroidal fields cannot be generated.

The above equations describing the evolution of magnetic fields inside the disk, must be supplemented by equations for the behaviour of the magnetic field outside the disk. Usually, vacuum conditions are assumed in the exterior region, i.e. no currents are flowing in the exterior region. If the magnetic fields are, however, loaded with disk plasma, the poloidal field structure has to be taken as a solution of the Grad-Shafranov equation (see later on), and currents are allowed to circulate between the disk surface and the wind region. This coupled 2D problem is quite complicated and has not yet been solved in the literature (for 1D solutions, see Ferreira, 1997).

For rapidly rotating disks, this dynamical system evolves naturally into a quadrupolar structure (Camenzind, 1990; Stepinski \& Levi, 1991; Rüdiger et al., 1995), quite in analogy to the time evolution of galactic magnetic fields. It is quite important for the stability of the magnetic fields that accretion disks are highly diffusive. Simulations based on ideal MHD equations are not suitable to test the time evolution of magnetic fields in disks. Outflows driven by such configurations could stabilize these structures. But at the moment there is no way to couple numerically a diffusive disk with an ideal MHD flow exterior to the disk. Disks have extremely low Reynolds numbers, outflows high Reynolds numbers.

Dragging of magnetic fields in accretion disks If the outer boundary conditions are set to bring in continuously dipolar flux, the inner disk could be forced to a dipolar configuration, $\Psi=\Psi_{d}+\Psi_{\infty}$ with $\Psi_{\infty}=R^{2} B_{\infty} / 2$ (Reyes-Ruiz \& Stepinski, 1996). This adds a source term to the diffusion equation for the poloidal flux generated in the disk

$$
\frac{\partial \Psi_{d}}{\partial t}+\vec{v} \cdot \nabla \Psi_{d}-\eta R^{2} \nabla \cdot\left[\frac{1}{R^{2}} \nabla \Psi_{d}\right]=-v_{R} R B_{\infty}
$$


The evolution of the field in the disk is essentially determined by the magnetic Prandtl number $P_{m}=\eta / \nu \simeq 1$ (Reyes-Ruiz \& Stepinski, 1996). Significant bending of the field lines only occurs for small Prandtl numbers, $P_{m} \simeq 0.01$. Only in this case the field lines are bent sufficiently to allow the development of a centrifugally driven wind (Blandford \& Payne, 1982). The toroidal field in the disk depends inversely on the magnetic diffusivity. These results may change, if dynamo action is taken into account for the internal evolution.

These pure disk configurations have another drawback. Magnetic fields from the inner disk are rapidly collimated into a cylindrical shape (Ouyed et al., 1997). The resulting jet radii are generally too small.

\subsection{JETS AS INDUCED STELLAR WINDS}

Fields from disks can be flared up to larger radii, provided there is some extra pressure along the axis. The most natural source of such a pressure is an additional magnetosphere built up by the central star itself. Such configurations have been discussed by the present author for the extragalactic case already in 1986 (Camenzind, 1986a) and applied to protostellar outflows in 1990 (Camenzind, 1990). A somewhat similar model has been discussed by Shu et al. in 1994. Personally, I think that only the existence of such a central pressure can provide the observed large collimation radii.

The strong differential rotation of accretion disks is responsible for the excitation of quadrupolar modes in disks. Since differential rotation is probably suppressed in protostellar objects (Rüdiger, private communication), the original dipolar structure left over from the collapse process, could be stabilized over long time scales. This is still an open question, but this mechanism would provide a different scenario for jet formation (Camenzind, 1990). Initially, we thought that the interaction between the stellar magnetosphere and the surrounding disk could drive the outflows (see also Fendt et al., 1995; Goodson et al., 1997).

Such outflows could, however, also be driven by the central object itself. This idea has a long history and requires that the central object carries an axisymmetric magnetosphere. Jet outflows occur in magnetized astrophysical systems whenever they provide a perfect axisymmetry. Real threedimensional objects such as pulsars and Weak-Line $\mathrm{T}$ Tauri stars never show the existence of collimated plasma flows.

Convection and shear in the protostellar cores are also driving forces for a stellar dynamo. Rapidly rotating protostars have sufficient energy to build up large-scale magnetic fields which will be strongly interacting with its surrounding accretion disk. Neglecting meridional circulations, the 
stellar dynamo equations have a much simpler form than the disk dynamo

$$
\begin{aligned}
\frac{\partial \Psi}{\partial t}-\eta_{*} R^{2} \nabla \cdot\left\{\frac{1}{R^{2}} \nabla \Psi\right\} & =R_{\alpha} \alpha_{*} I \\
\frac{\partial I}{\partial t}-R^{2} \nabla \cdot\left\{\frac{\bar{\eta}_{*}}{R^{2}} \nabla I\right\} & =R_{\Omega} R^{2} \vec{B}_{p} \cdot \nabla \bar{\Omega}-\frac{I}{\tau_{\text {loss }}} f\left(B / B_{\text {crit }}\right) .
\end{aligned}
$$

Here $R_{\alpha}=\alpha_{*} R_{*} / \eta_{*}, R_{\Omega}=\Omega_{*} R_{*}^{2} / \eta_{*}$, and $\mathcal{P}=R_{\alpha} R_{\Omega}$ is the dynamo number. Radii are given in units of the stellar radius $R_{*}$, time in units of $R_{*}^{2} / \eta_{*}$, and the toroidal field $B_{\phi}$ in units of $B_{*}$ which depends on the stellar density

$$
B_{*}=\sqrt{4 \pi \rho_{*}} \frac{\eta_{*}}{R_{*}}
$$

The typical scale of the $\alpha$-effect is as usually given by

$$
\alpha \simeq\left\{\begin{array}{rll}
l(z) \Omega_{*}(z) & : & l \Omega_{*} / v_{c} \ll 1 \\
v_{c}(z) & : & l \Omega_{*} / v_{c} \gg 1
\end{array}\right\}
$$

$v_{c}$ is the convective velocity in the star. Convective velocity and Rossby number $R_{o}=\left(\Omega_{*} \tau_{c}\right)^{-1}$ follow from the energy transport in the star

$$
L_{*}=\epsilon_{c} M_{*} v_{c}^{3} / R_{*},
$$

and therefore

$$
\begin{aligned}
v_{c} \simeq & 40 \mathrm{~m} \mathrm{~s}^{-1}\left(\epsilon_{c} / 30\right)^{-1 / 3}\left(L_{*} / L_{\odot}\right)^{1 / 3} \\
& \left(M_{*} / M_{\odot}\right)^{-1 / 3}\left(R_{*} / R_{\odot}\right)^{1 / 3}
\end{aligned}
$$

This would correspond to a mean Rossby number in the star

$$
\begin{aligned}
R_{o}= & 1.5 \times 10^{-3} \omega^{-1}\left(L_{*} / 4 L_{\odot}\right)^{1 / 3}\left(\epsilon_{c} / 30\right)^{2 / 3} \\
& \left(M_{*} / M_{\odot}\right)^{-5 / 6}\left(R_{*} / 3 R_{\odot}\right)^{5 / 6}
\end{aligned}
$$

The helicity $\alpha$ is a measure of the lack of symmetry of the convective flow. For small Rossby numbers the correct expression is given by

$$
\alpha=\alpha_{0} \bar{\alpha}(r, \theta) \quad, \quad \alpha_{0} \simeq L_{c} v_{c} / H_{P}=\gamma v_{c} \quad, \quad \gamma<1 .
$$

$H_{P}$ is the pressure scale height. In this kinematic dynamo scheme, magnetic fields are found to grow exponentially, or to decay exponentially. Nonlinear feedback between strong magnetic fields and turbulence suggests however the existence of a critical field strength $B_{\text {crit }}$ for the onset of toroidal flux loss and quenching of the $\alpha$-effect

$$
\alpha=\alpha_{0} \bar{\alpha}(r, \theta) q\left(B / B_{\text {crit }}\right),
$$


where $q$ is a dimensionless function which tends to zero for $B^{2}>B_{\text {crit }}^{2}$. Since this critical field strength is the natural scale for the toroidal field, the dynamo equation for the poloidal flux also provides the units $\Psi_{0}$ for $\Psi$

$$
\Psi_{0}=\frac{R_{\mathrm{in}}^{3} B_{\text {crit }} \alpha_{0}}{\eta_{*}}=3 \frac{R_{\mathrm{in}}}{L_{c}} R_{\mathrm{in}}^{2} B_{\text {crit }} \gamma R_{o}^{-p} .
$$

$R_{\text {in }}$ is the inner radius for the convective zone of the protostar.

In these natural units, the dynamo equations only contain one essential coupling constant. This is the dynamo number $\mathcal{P}$, defined by

$$
\mathcal{P}=\frac{R_{\mathrm{in}}^{3} \Omega_{\mathrm{in}} \alpha_{0}}{\eta_{*}^{2}}=\frac{9}{P_{m}}\left(\frac{R_{\mathrm{in}}}{L_{c}}\right)^{2} \frac{R_{\mathrm{in}} \Omega_{\mathrm{in}}}{v_{c}} \frac{\alpha_{0}}{v_{c}} R_{o}^{-2 p} .
$$

Using the scaling relations for rapidly rotating stars, we get

$$
\mathcal{P} \simeq 4 \times 10^{9} \gamma \omega^{3}\left(\frac{R_{\text {in }}}{L_{c}}\right)^{2} \frac{R_{\text {in }}}{0.1 R_{*}} \quad, \quad \omega=\frac{\Omega_{*}}{\Omega_{K}\left(R_{*}\right)} .
$$

Here, we used the magnetic Prandtl number $P_{m}, \eta_{*}=P_{m} \nu_{*}, P_{m} \simeq 1$. The corresponding dynamo number is fantastically high for rapidly rotating convective stars and scales as $\mathcal{P} \propto \omega^{3}$ with the angular velocity. But even for moderately rotating $\mathrm{T}$ Tauri stars, $\omega \simeq 0.1$, we still find extremely high dynamo numbers, $\mathcal{P}_{\text {TTS }} \simeq 4 \times 10^{5}$, while the Sun has the dynamo number $\mathcal{P}_{\odot} \simeq 1000-2000$ as obtained from other estimates. This extremely high dynamo number for protostellar objects is the second difference between solar-like stars (see e.g. Küker et al. 1996) and pre-main sequence objects. It makes any scaling between solar phenomena and protostellar properties completely obsolete. The dynamo number is responsible for the characteristic mode excited in the star. The structure which evolves under high dynamo numbers will crucially depend on the nonlinear saturation process. No models have been calculated for this domain of dynamo numbers. So we can only speculate on the resulting magnetic structure.

One of the crucial quantities is the critical field strength where saturation occurs. There are various ways to derive this quantity. In a first attempt, the critical field strength essentially follows from the condition that convection must be super-Alfvénic, i.e. the convective energy density should supercede the magnetic energy density,

$$
B_{\text {crit }} \simeq v_{A} \sqrt{4 \pi \rho_{*}}=\frac{v_{c}}{M_{T}} \sqrt{4 \pi \rho_{*}} .
$$

In natural units we obtain

$$
B_{\text {crit }} \simeq 10 \mathrm{kG} \frac{0.3}{M_{T}}\left(\frac{L_{*}}{4 L_{\odot}}\right)^{1 / 3}\left(\frac{M}{M_{\odot}}\right)^{1 / 6} .
$$


The typical toroidal field strength built up by dynamo action in a protostellar convection zone is of the order of 10 kilo-Gauss. This critical field strength is independent of the rotation period of the star, and is therefore also the typical field strength expected in the lower convection zone of the Sun (Küker et al., 1996). From the expression (13) we then get the poloidal magnetic flux in the interior of the star

$$
\Psi_{0} \simeq 3 \times 10^{26} \mathrm{G} \mathrm{cm}^{2} \frac{\gamma \omega}{0.1} \frac{B_{\text {crit }}}{10 \mathrm{kG}} .
$$

From this we find the typical poloidal field strength at the surface of the star

$$
B_{*} \simeq \Psi_{0} / R_{*}^{2} \simeq 6 \mathrm{kG} \frac{\omega \gamma}{0.1} \frac{B_{\text {crit }}}{10 \mathrm{kG}},
$$

which would be about 2 kilo-Gauss for a protostar rotating with 5 days. According to this scaling law, rapidly rotating protostellar cores would have poloidal fields at the surface of the order of 10 kilo-Gauss, while $\mathrm{T}$ Tauri stars with $\omega \simeq 0.1$ would still have magnetic fields of the order of one kilo-Gauss at their surface.

\section{Rotation and Jet Energy}

Rotation is the ultimate energy source for magnetically driven winds. Though the solar wind is magnetized, it is not magnetically driven. Winds ejected from rapidly rotating stars and disks are, however, magnetically driven. When a solar type star reaches the main sequence with a rotation rate $\Omega_{*} \simeq 20 \Omega_{\odot}$ at an age of 50 million years, then the thermally driven solar wind changes to a completely magnetically and rotationally driven MHD wind (MacGregor, 1996). Historically, these winds have been discussed as spherically symmetric winds in the equatorial plane of a star that rotates uniformly with angular velocity $\Omega_{*}$ (Weber \& Davis, 1967; Belcher \& MacGregor, 1976; Charbonneau, 1995). Fully 2D axisymmetric winds have been discussed so far only in the self-similar approach.

\subsection{WHAT DRIVES THE ACCELERATION?}

When a rotating body carries an axisymmetric magnetosphere, rotation induces electric fields $\vec{E}_{\perp}$ over the unipolar induction such that the magnetic surfaces become equipotential surfaces. These electric fields together with the toroidal magnetic fields $\vec{B}_{T}$ give rise to a Poynting flux (Camenzind, 1986a)

$$
\vec{P}_{p}=\frac{c}{4 \pi} \vec{E}_{\perp} \wedge \vec{B}_{T}
$$


This can be written in the form, $I=-c R B_{\phi} / 2$,

$$
\vec{P}_{p}=\frac{\Omega_{*} I}{2 \pi c} \vec{B}_{p}
$$

When integrating this Poynting flux over the entire surface of the central object, we end up with a magnetic luminosity

$$
L_{\mathrm{mag}}=\frac{1}{c} \Omega_{*} I_{*} \Psi_{*},
$$

depending on the rotational state of the object, the total magnetic flux $\Psi_{*}$ carried by the magnetosphere and the total current $I_{*}$ driven by the rotation of the object. This magnetic luminosity is a new source of energy which is usually neglected in the energy budgets. It is however an important source of energy for strongly magnetized and rapidly rotating objects.

Young stellar objects at the age of a few hundred thousand years are observed to rotate faster than the Sun, $\Omega_{*} \simeq 20 \Omega_{\odot}$ with $\Omega_{\odot}=3 \times 10^{-6}$ $\operatorname{rad~} \mathrm{s}^{-1}$, and to carry a stronger magnetosphere. Field strengths of the order of 1000 Gauss are consistent with observations. This corresponds to a magnetic luminosity of the rotating star

$$
L_{\mathrm{mag}}=0.1 L_{\odot} \frac{\Psi_{*}}{10^{25} \mathrm{Gauss} \mathrm{cm}^{2}} \frac{I_{*}}{10^{14} \mathrm{Amps}} \frac{\text { days }}{P_{*}}
$$

with a magnetic flux given in units of $10^{25}$ Gauss $\mathrm{cm}^{2}$ and a current of $10^{14}$ Amperes. This is some fraction of the bolometric luminosity of low-mass stellar objects, but completely negligible in the case of the Sun due to its low mean magnetic field and its low rotation rate. For young stellar objects, the magnetic luminosity is close to the kinetic luminosity found in the jets of these objects

$$
L_{\mathrm{jet}}=0.1 L_{\odot} \frac{\dot{M}_{j}}{10^{-8} M_{\odot} y r^{-1}}\left(\frac{v_{j}}{300 \mathrm{~km} \mathrm{~s}^{-1}}\right)^{2} .
$$

The primary energy source driving the outflows can be either the protostellar rotation or the rotational energy stored in a surrounding disk. Protostars formed out of rotating molecular clouds will be born as rapid rotators. The rotational energy stored in the rapidly rotating protostellar object

$$
E_{\mathrm{rot}}=\frac{1}{2} k^{2} M_{*} R_{*}^{2} \Omega_{*}^{2}=4 \times 10^{46} \mathrm{erg} \frac{5 R_{\odot}}{R_{*}}\left(\frac{M_{*}}{M_{\odot}}\right)^{2}\left(\frac{P_{K}}{P_{*}}\right)^{2}
$$


is in fact of the order of the energy stored in the molecular outflows. This is a considerably energy, when it is dissipated on a time-scale of less than 100.000 years

$$
L_{\mathrm{rot}}=\frac{E_{\mathrm{rot}}}{\tau_{j}} \simeq 1.0 L_{\odot} \frac{10^{5} \mathrm{yr}}{\tau_{j}}\left(\frac{M_{*}}{M_{\odot}}\right)^{2}\left(\frac{3 P_{K}}{P_{*}}\right)^{2} .
$$

The critical period $P_{K}$ for a $1 M_{\odot}$ object would be about half a day. Since it scales quadratically with the mass, it can easily explain the observed mechanical luminosities (Lada, 1985). That this short time-scale is not unrealistic, follows from the braking time of a magnetized wind

$$
\tau_{m}=\frac{J_{*}}{d J_{*} / d t} \simeq k^{2}\left(\frac{R_{*}}{R_{A}}\right)^{2} \frac{M_{*}}{\dot{M}_{j}} \simeq 10^{5} \mathrm{yr}
$$

for mass-loss rates of the order of $\dot{M}_{j} \simeq 10^{-8} M_{\odot} \mathrm{yr}^{-1}$. Rapidly rotating protostars have in fact sufficient energy to drive this magnetic luminosity, disk accretion is not necessarily involved.

\subsection{THE LIGHT CYLINDER OF RAPIDLY ROTATING OBJECTS}

In the theory of rotating magnetospheres (as for pulsars e.g.), the appearance of the light cylinder is crucial. For protostellar objects, we find a light cylinder radius $R_{L}=c / \Omega_{*}$ given by

$$
R_{L}=\frac{c}{\Omega_{*}}=100 \mathrm{AU} \frac{P_{*}}{3 \text { days }} .
$$

This radius is interestingly close to the observed collimation radii. Once a magnetosphere extends beyond the light cylinder, Newtonian MHD becomes obsolete and has to be replaced by the special relativistic one (Camenzind, 1986a). The deeper reason for this is the existence of electric fields which can no longer be neglected in modelling the magnetosphere (Camenzind, 1987; 1989; Appl \& Camenzind, 1993a,b; Fendt et al., 1995). They contribute to the forces at the same level as magnetic fields do. Electric fields can only be neglected, if the jet radii are much smaller than one hundred AU.

\subsection{PLASMA MOTION ALONG MAGNETIC FLUX-TUBES AND PLASMA DIAGNOSTICS}

In ideal MHD, plasma injected at the foot points of magnetic flux tubes moves along these flux surfaces. The theory of axisymmetric and stationary plasma motion is well developed and will not be repeated here (for 
the Newtonian theory, see e.g. Heyvaerts \& Norman, 1996; for the general theory including electric fields and gravitational forces, see Camenzind, 1996). Applications to 2D protostellar magnetospheres are discussed in Fendt \& Camenzind (1996), and Paatz \& Camenzind (1996a). The particular form of these outflows is used to explain the origin of the low-velocity and high-velocity components of forbidden emission lines (Paatz \& Camenzind, 1996b). The differences between their profiles follow from their different critical densities and the rotational properties of the wind in the formation region.

\section{Collimation of MHD Jets: Cylindrical Pinch Solutions}

The collimation of magnetized winds into a conical outflow is very similar to the plasma confinement in a $z$-pinch. The equilibrium condition for a non-rotating axisymmetric current carrying pinch configuration has to be generalized to include various relativistic effects (Appl \& Camenzind 1993a,b; Fendt et al., 1995)

$$
\begin{aligned}
\kappa \frac{B_{p}^{2}}{4 \pi}\left(1-M^{2}-x^{2}\right) & =\left(1-x^{2}\right) \nabla_{\perp} \frac{B_{p}^{2}}{8 \pi}+\nabla_{\perp} \frac{B_{\phi}^{2}}{8 \pi}+\nabla_{\perp} P \\
-\frac{B_{p}^{2} \Omega_{*}}{4 \pi c^{2}} \nabla_{\perp}\left(\Omega_{*} R^{2}\right) & +\left(\frac{\rho j^{2}}{R^{3}}-\frac{I^{2}}{4 \pi R^{3}}\right)\left(-\nabla_{\perp} R\right) .
\end{aligned}
$$

Pressure gradients acting perpendicular to the magnetic surfaces are in equilibrium with electric forces, centrifugal forces, pinch forces produced by poloidal currents, as well as with curvature forces expressed at the left hand side. This latter force is modified by the Mach number $M$ and the light cylinder, $x=R / R_{L}$, and $j=R^{2} \Omega$.

We discuss in the following the solutions for a cylindrical pinch, where the curvature forces vanish, $\kappa=0$. In this case, the equilibrium condition reduces to its one-dimensional form

$$
\left(1-x^{2}\right) \frac{d}{d R} \frac{B_{p}^{2}}{8 \pi}+\frac{d}{d R} \frac{B_{\phi}^{2}}{8 \pi}+\frac{d P}{d R}-\frac{B_{p}^{2} R}{2 \pi R_{L}^{2}}-\left(\frac{\rho j^{2}}{R^{3}}-\frac{I^{2}}{4 \pi R^{3}}\right)=0 .
$$

The pressure of the toroidal field and the pinch force can be combined into one single expression. In addition, we normalize radii in units of the light cylinder $R_{L}, x=R / R_{L}$, and the poloidal magnetic field in units of its value on the central axis, $B_{0}, y=B_{p}^{2} / B_{0}^{2}$,

$$
\left(1-x^{2}\right) \frac{d y}{d x}-4 x y+\frac{1}{x^{2}} \frac{d I^{2}}{d x}+\frac{8 \pi}{B_{0}^{2}} \frac{d P}{d x}-\frac{8 \pi \rho}{R_{L}^{2} B_{0}^{2}} \frac{j^{2}}{x^{3}}=0 .
$$


Electric fields, $E_{\perp}=\left(R / R_{L}\right) B_{p}$, due to the rotation of the magnetic surfaces modify the classical pinch equilibrium in two ways. First, the equation has a singular point at the light cylinder, and secondly, the additional term $4 x y$ is crucial for the form of the equilibrium. In contrast to force-free models (Appl \& Camenzind, 1993b), the specific angular momentum $j$ of the plasma and the poloidal current $I$ are not arbitrary, but follow from the MHD wind theory (Camenzind, 1996).

We only discuss solutions of the relativistic pinch equilibrium neglecting the influence of the centrifugal force. In this case the equilibrium is determined by the condition

$$
\left(1-x^{2}\right) \frac{d y}{d x}-4 x y+\frac{1}{x^{2}} \frac{d I^{2}}{d x}+\frac{8 \pi}{B_{0}^{2}} \frac{d P}{d x}=0 .
$$

The toroidal current follows to a good approximation

$$
I \simeq-\Omega_{*}\left(R^{2} B_{p}\right) \frac{1}{\beta_{p}}=-c B_{0} R_{L}\left(x^{2} \sqrt{y}\right) \frac{1}{\beta_{p}}
$$

and we prescribe the plasma pressure in terms of $P=\beta B_{p}^{2} / 8 \pi$ with a constant plasma beta. This leads finally to the equation, $\gamma_{p}=1 / \sqrt{1-\beta_{p}^{2}}$,

$$
\left(1+\beta+\frac{x^{2}}{\gamma_{p}^{2} \beta_{p}^{2}}\right) \frac{d y}{d x}+\frac{4}{\gamma_{p}^{2} \beta_{p}^{2}} x y=0 .
$$

This homogeneous equation has the remarkably simple solution

$$
y(x)=\frac{C}{\left(1+\beta+x^{2} / x_{c}^{2}\right)^{2}}
$$

with

$$
x_{c}=\gamma_{p} \beta_{p} \simeq 10^{-3} \quad, \quad R_{c}=\gamma_{p} \beta_{p} R_{L} \simeq 10^{-3} R_{L}
$$

as the core radius of the jet with $\beta_{p} \simeq 10^{-3}$ and $\gamma_{p}=1$ for protostellar outflows. Inside the core, given by the light cylinder radius and the poloidal velocity $\beta_{p}$, the poloidal magnetic field is essentially homogeneous. It decays as a monopole field outside the core, $B_{p} \propto 1 / R^{2}$ for $R \gg R_{c}$,

$$
B_{p}=\frac{B_{0}}{1+\beta+R^{2} / R_{c}^{2}}
$$

This is not unexpected, since the jet is formed by a supermagnetosonic wind. The current function $I$ increases quadratically inside the core and saturates towards a constant value outside the core, $I_{\infty} \simeq c B_{0} R_{L} \beta_{p}$. This 
is exactly the structure of the force-free solutions found previously (Appl \& Camenzind, 1993b).

MHD jets always have a core-envelope structure, along the axis the magnetic field is predominantly longitudinal, beyond the core it becomes dominated by the toroidal component. The light cylinder $R_{L} \simeq 100 \mathrm{AU}$ is the basic scale for the jets, the core is extremely small, $R_{c} \simeq 10 R_{*}$.

\section{Propagation of MHD Jets: Knots as Internal Pinch Modes}

Protostellar jets differ from extragalactic jets in that radiative cooling is dynamically important. Protostellar jets are observable because of their radiative losses. HD simulations are parametrized by three dimensionless quantities

- the sonic Mach number, $M_{s} \geq 20$;

- the density contrast $\eta=1-10$, jet density to ambient density;

- cooling length $\chi=$ ratio between cooling length and jet radius.

An adiabatic jet has $\chi=\infty$ while for an isothermal jet $\chi=0$, realistic values are $\chi \simeq 1-10$.

Many protostellar jets have impressive arrays of emission knots in the inner part of the jet. Such knots can occur from overpressured jets. Reconfinement shocks are formed with emission knots behind them. But, the knot spacing is too large for hydro-jets $(\simeq 10)$ and the knots proper motion is predicted to be zero, whereas they are observed to move with $20-100 \%$ of the jet speed.

The inclusion of magnetic fields into jet simulations has various effects. No MHD simulations have been done including radiative cooling. From adiabatic simulations it is known that magnetic fields have various effects on the flow structure and the stability of jets (Clark et al., 1990; Kössl et al., 1990; Lind et al., 1989). Compared to hydrodynamic models, the bow shock is in general further separated from the end of the jet due to magnetic pressure. This effect depends dramatically on the magnetic field structure and the magnetic diffusivity which is used in the simulation. An important effect is due to the reduction of the essential Mach number which is now the magnetosonic Mach number, $M_{\mathrm{MS}} \simeq 2-3$. The sonic speed is no longer the characteristic speed in magnetized jets. Third, KelvinHelmholtz instabilities are reduced by magnetic field tension. And finally, in 3D simulations, helical motions appear which are reminiscent of HH46/47.

Protostellar MHD jets are low $\beta$ flows

$$
\beta=\frac{P_{\text {gas }}}{B^{2} / 8 \pi} \simeq 0.1-0.01
$$



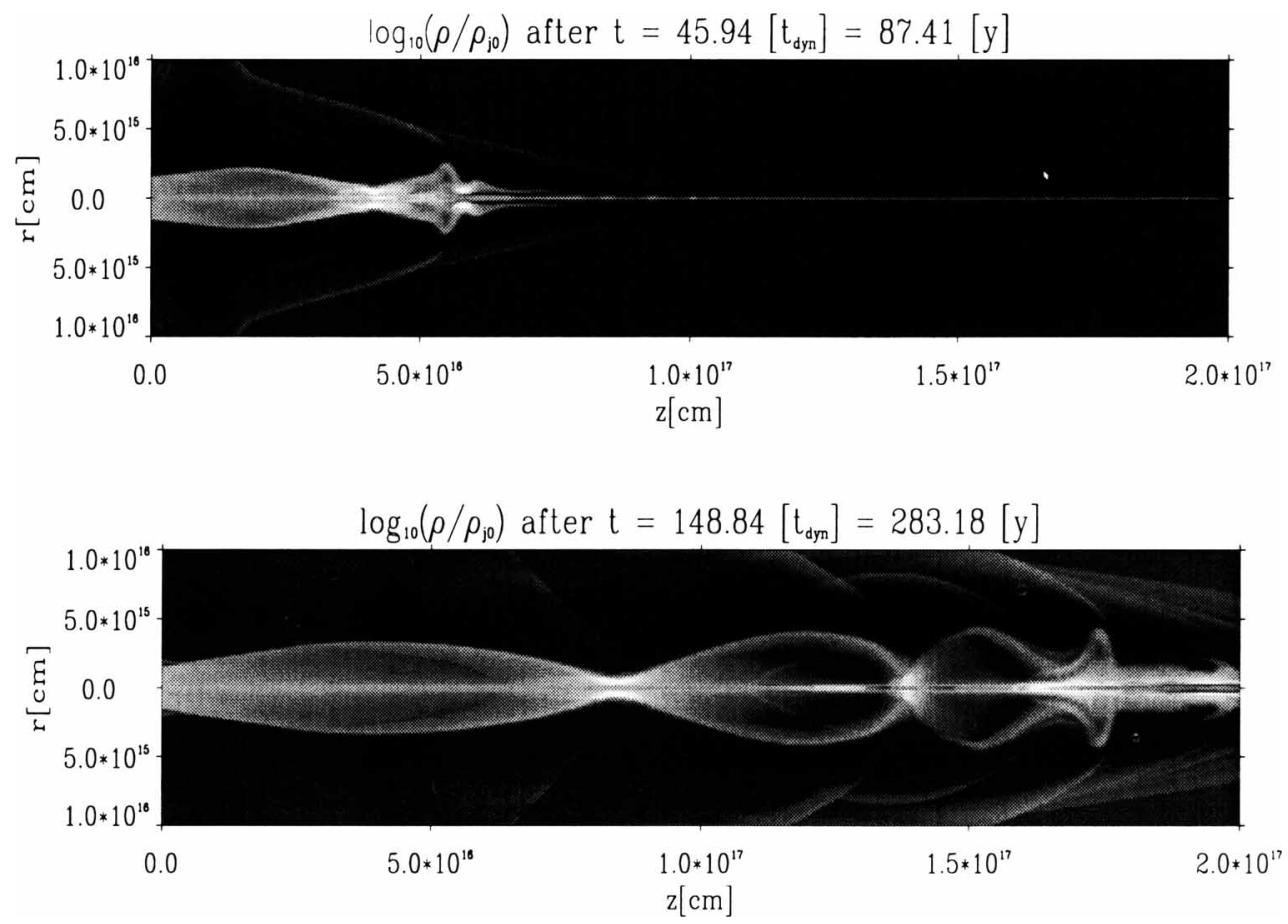

Figure 2. The time-evolution of a core-envelope jet with a monopolar poloidal magnetic field and a toroidal field initially constrained to some region (Thiele 1997, LSW Heidelberg). The beam is ten times overdense with respect to the ambient medium, the density is shown as grey scale. The inital beam radius is $100 \mathrm{AU}$, the jet has propagated for 87 years (upper panel) and for 283 years (lower panel) from left to right.

and have strong toroidal magnetic fields, $B_{\phi} \gg B_{p}$ (the observable part of the jet is formed by the envelope structure, the core is not resolvable). Under these conditions, pinch modes are excited (Fig. 2), the strong toroidal field focuses the flow towards the axis so that a quasi-periodic structure appears.

These simulations demonstrate that axisymmetric knots are produced very close to the collimation zone on time scales given by the characteristic dynamical time

$$
\tau=\frac{R_{j}}{v_{j}} \simeq 0.5 \text { year } \frac{R_{j}}{50 \mathrm{AU}} \frac{300 \mathrm{~km} \mathrm{~s}^{-1}}{v_{j}} .
$$

They occur roughly on 10 dynamical time scales. Knot generation is therefore inherent to the dynamics of the jet itself, no episodic ejection is needed 
(see also Ouyed et al., 1997). These knots persist as coherent structures which propagate along the jet channel at a fraction of the jet speed. Investigations into the thermal structure of these knots will demonstrate the feasibility of this model for explaining the emission properties of the forbidden lines from these jets.

Obviously, there are multiple bow shocks on longer time scales, typically found at spacings of a few thousand years. These variations must be intrinsic to the central source or the surrounding accretion disk. These longer time scales could be related to magnetic transport in the disk, or some characteristic dynamo time scale of the central star.

\section{Conclusions}

MHD jet models for protostellar sources close a gap between jet formation scenarios and the propagation properties of young stellar jets. There is no doubt that protostellar outflows must be collimated by magnetic processes. One can therefore not avoid to treat the propagation of these outflows also within the framework of MHD. The main difference between hydro jets and MHD jets is the Mach number - MHD jets have low Mach numbers of order $2-3$, since the relevant signal speed is the fast magnetosonic speed, which is of the order of $100 \mathrm{~km} / \mathrm{s}$, and no longer the sound speed. This low Mach number together with the low plasma- $\beta$ makes them vulnerable to internal instabilities. Since the toroidal magnetic field is dominant in the outer parts of the jets, the pinch mode is naturally excited. These fields are extremely weak, less than a milli-Gauss. Pinch modes create oscillations that propagate as coherent structures along the jet and evolve probably into weak magnetosonic shocks. The emission knots can probably be associated with these structures.

The very origin of poloidal and toroidal magnetic fields in the jets is still under debate. Magnetized central objects are good candidates, since their energetics can explain the observed properties of the jets. The ultimate energy source behind these outflows is the rotational energy of the central star, or its surrounding disk.

Acknowledgements: Part of this work is supported by the Schwerpunktsprogramm Physik der Sternentstehung of the Deutsche Forschungsgemeinschaft. The time-dependent MHD simulations have been provided by Markus Thiele.

\section{References}

Appl, S., Camenzind, M.: 1992, A\&A 256, 354

Appl, S., Camenzind, M.: 1993a, A\&A 270, 71

Appl, S., Camenzind, M.: 1993b, A\&A 274, 699 
Blandford, R.D., Payne, D.G.: 1982, MNRAS 199, 883

Belcher, J.W., MacGregor, K.B.: 1976, ApJ 210, 498

Camenzind, M.: 1986a, A\&A 156, 137

Camenzind, M.: 1986b, A\&A 162, 32

Camenzind, M.: 1987, A\&A 184, 341

Camenzind, M.: 1989, in Accretion Disks and Magnetic Fields in Astrophysics, ed. G. Belvedere, Kluwer (Dordrecht), p. 129

Camenzind, M.: 1990, in Reviews of Modern Astronomy 3, ed. G. Klare, Springer-Verlag (Heidelberg), p. 234

Camenzind, M.: 1993, in The Jets of Radio Galaxies, eds. H.J. Röser \& K. Meisenheimer, Lecture Notes in Physics 421, Springer-Verlag (Heidelberg), p. 109

Camenzind, M. 1996, in Solar and Astrophysical Magnetohydrodynamic Flows, ed. K.C. Tsinganos, Kluwer (Dordrecht), p. 699

Camenzind, M., Lesch, H.: 1994, A\&A 284, 411

Charbonneau, P. 1995, ApJS 101, 309

Clark, D.A., Stone, J.M., Norman, M.L.: 1990, BAAS 22, 801

Fendt, C., Camenzind, M., Appl, S.: 1995, A\&A 300, 791

Fendt, C., Camenzind, M. 1996, A\&A 313, 591

Ferreira, J., Pelletier, G.: 1995, A\&A 295, 807

Ferreira, J.: 1997, A\&A, in press

Goodson, A.P., Winglee, R.M., Böhm, K.H.: 1997, ApJ, submitted

Hartmann, L., MacGregor, K.B.: 1982, ApJ 259, 180

Heyvaerts, J., Norman, C.A. 1996, in Solar and Astrophysical Magnetohydrodynamic Flows, ed. K.C. Tsinganos, Kluwer (Dordrecht), p. 459

Kössl, D., Müller, E., Hillebrandt, W.: 1990, A\&A 229, 378, 397

Küker, M., Rüdiger, G., Pipin, V.V.: 1996, A\&A 312, 615

Lada, C.J.: 1985, AAR\&A 23, 267

Lada, C.J., Fich, M.: 1996, ApJ 459, 638

Lind, K., Payne, D.G., Meier, D.L., Blandford, R.D.: 1989, ApJ 344, 89

MacGregor, K.B.: 1996, in Solar and Astrophysical Magnetohydrodynamic Flows, ed. K.C. Tsinganos, Kluwer (Dordrecht), p. 301

McCaughrean, M.J., Rayner, J.T., Zinnecker, H.: 1994, ApJL 436, L189

Ouyed, R., Pudritz, R.E., Stone, J.M.: 1997, Nature 385, 409

Paatz, G., Camenzind, M.: 1996a, A\&A 308, 77

Paatz, G., Camenzind, M.: 1996b, Astro. Lett. and Communications 34, 315

Pelletier, G., Pudritz, R.E.: 1992, ApJ 394, 117

Pudritz, R.E., Norman, C.A.: 1986, ApJ 301, 571

Ray, T.P., Mundt, R., Dyson, J.E., Falle, S.A.E.G., Raga, A.C.: 1996, ApJL 468, L103

Reyes-Ruiz, M., Stepinski, T.F.: 1996, ApJ 459, 653

Rüdiger, G., Elstner, D., Stepinski, T.F.: 1995, A\&A 298, 934

Shu, F.H., Najita, J., Wilkin, F., Ruden, S.P., Lizano, S.: 1994, ApJ 429, 781

Stapelfeldt, K.R., et al.: 1995, ApJ 449, 888

Stepinski, T.F., Levy, E.H.: 1991, ApJ 379, 343

Stone, J.M., Norman, M.L.: 1993, ApJ 413, 210

Suttner, G., Smith, M.D., Yorke, H.W., Zinnecker, H.: 1997, A\&A, in press

Weber, E.J., Davis, L.: 1967, ApJ 148, 217

Zinnecker, H., McCaughrean, M.J., Rayner, J.T.: 1996, in Disks and Outflows around Young Stars, eds. S. Beckwith, J. Staude, A. Quetz and A. Natta, Lecture Notes in Phys. 465, (Heidelberg, Springer-Verlag), p. 236 